\title{
Historein
}

Vol 15, No 2 (2015)

Historein 15/2 (2015)

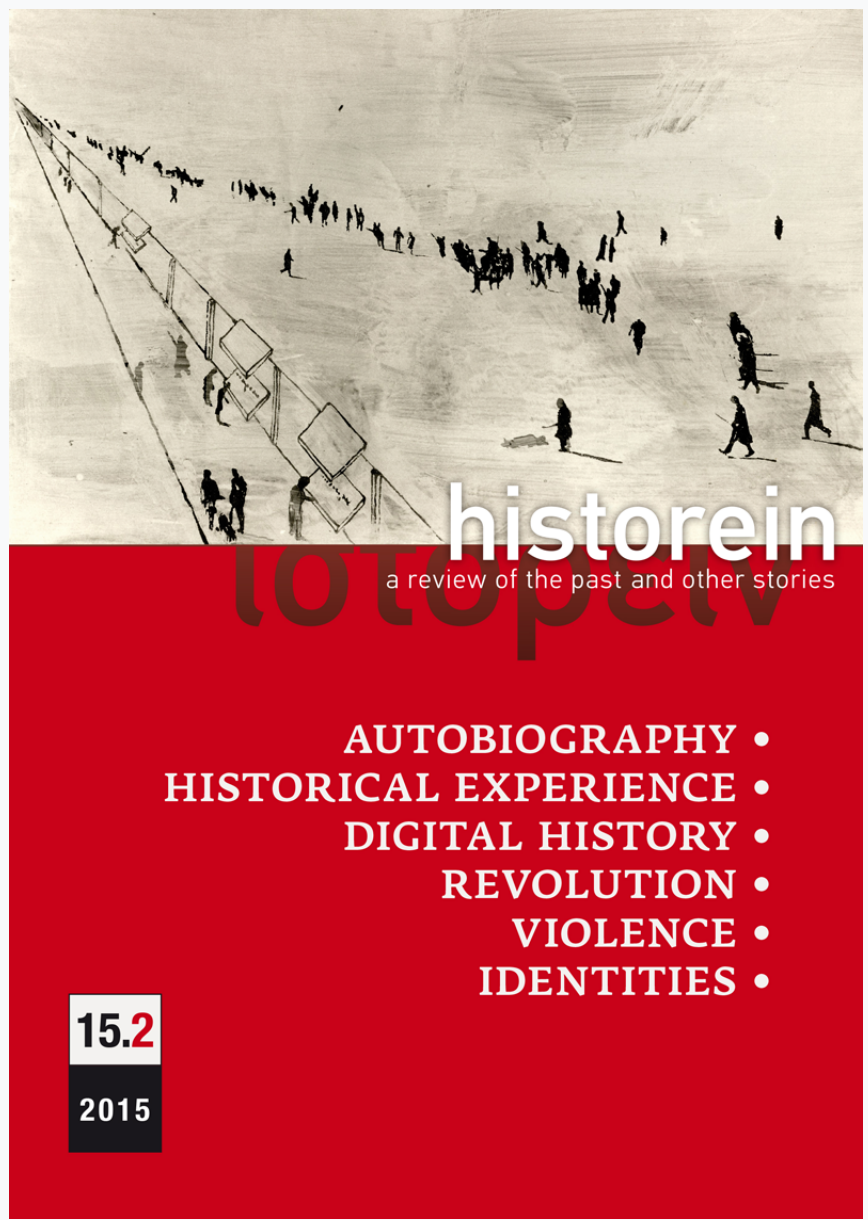

\section{Review of Constantin Irodotou's, Des utopies} sadiennes

Georges Faraklas

doi: $10.12681 /$ historein.8701

Copyright ( 2015 , Georges Faraklas

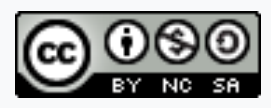

This work is licensed under a Creative Commons Attribution-NonCommercialShareAlike 4.0.

To cite this article:

Faraklas, G. (2016). Review of Constantin Irodotou's, Des utopies sadiennes. Historein, 15(2), 99-102. https://doi.org/10.12681/historein.8701 
the editors chosen to have an open call to embellish this undertaking with more papers, instead of limiting it to the ones delivered (or selected but never actually presented) at the conference (such as the extended researches by photography historian Hercules Papaioannou on the Greek landscape, and those of visual sociologists Gregory Paschalidis on Greek iconic photographs and Yannis Skarpelos on foreign photographers representing Greece), this volume would be an even more significant addition to the curriculum.

\section{NOTE}

1 See, for example, Alkis A. Xanthakis, lotopía

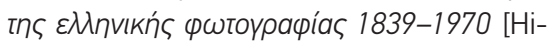
story of Greek Photography, 1839-1960] (Athens: Kastaniotis, 1981); John Stathatos, Image and Icon: The New Greek Photography, 1975-1995 (Thessaloniki: Hellenic Ministry of Culture/Macedonian Museum of Contemporary Art, 1997); Kostas loannidis, ¿úyxpovn

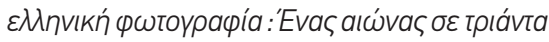
xpóvia [Contemporary Greek photography: a century in 30 years] (Athens: Futura, 2008).

\section{Constantin Irodotou}

\section{Des utopies sadiennes}

Paris: L'Harmattan, 2015. 133 pp.

\section{Georges Faraklas}

Panteion University, Athens

L'ouvrage de Constantin Irodotou, Des utopies sadiennes, version remaniée d'une partie de sa thèse de philosophie Sade / Fourier: utopie et sexualité (Paris VIII, 2014), s'emploie à repenser le rapport entre histoire et fiction à partir de certaines œuvres de Sade, notamment des deux fictions opposées que l'on trouve dans Aline et Valcour, roman épistolaire écrit à la Bastille avant la Révolution, et publié en 1795: «Butua» est un royaume qui promeut la sexualité extra-conjugale tous azimuts et le crime, "Tamoé» un île d'où le crime est absent et où la sexualité est confinée au cadre marital.' À Butua la population décroît, à Tamoé, elle croît. Or, il est étonnant de constater, avec Irodotou, que la relation de Butua est présentée comme une enquête et, partant, comme historique, tandis que celle de Tamoé est dite chimérique. Il y a là de quoi s'étonner, car l'une et l'autre relèvent bien de la fiction. Quel est le sens d'une telle «historicité»? En quoi une fiction est-elle historique? Telle est la question que se pose Irodotou (p. 68). ${ }^{2}$ Il semble nous suggérer deux réponses, de valeur inégale. L'une serait que l'on peut désigner comme «historique» le récit de ce qui peut avoir existé, même si les événements décrits sont fictifs. L'autre consisterait à dire qu'est «historique» ce qui fait évoluer la nature, même sans pouvoir la changer. Si, selon la première réponse, une fiction historique est une fiction réaliste, ce qui réduit l' «historicité» à un avatar du «réalisme», le second type de réponse, à juste titre favorisé par Irodotou, est beaucoup plus riche d'enseignements. 
Comme Irodotou nous le rappelle, Sade s'est essayé au genre historique, notamment dans son Histoire secrète d'Isabelle de Bavière, achevée peu avant sa mort, en 1814 (p. 55). Selon Sade, l'épouse de Charles VI a si bien assouvi sa soif de puissance et de luxure, qu'on lui peut «raisonnablement» imputer la totalité des crimes commis sous ce règne (p. 60). Or, Sade se défend de décrire cette apothéose du crime par goût. S'il le fait, dit-il, c'est que l'historien ne doit rien omettre des crimes qui ont eu lieu, au rebours du romancier, qui, libre de nous présenter les hommes «tels qu'ils devraient être», est «dispensé de nous tracer les crimes». ${ }^{3}$

Or, chacun le sait, Sade romancier ne s'est guère privé de dépeindre des crimes (p. 58). Mais cela ne veut pas dire qu'il cherche ici à nous tromper. Sans doute accordera-t-on qu'il ment quand il traite de «calomnie» l'attribution à lui de la paternité de Justine, ouvrage publié anonymement qui dépeint des crimes, sauf à supposer qu'il souffre de dédoublement de la personnalité, ${ }^{4}$ ce qui reviendrait à adopter la thèse, peu crédible, de la maladie mentale. ${ }^{5}$ Mais on a tout lieu de penser que l'opposition de l'historique et du romanesque ici évoquée se situe à un niveau étranger au mensonge, si l'on remarque - avec Irodotou - que cette opposition recoupe celle de l'historique et du chimérique dans Aline et Valcour.

En effet, si le romancier est «dispensé» de décrire les crimes, cela ne lui est pas impossible, et si le mal est historique, s'il appartient à l'être, tandis que seul le roman peut narrer ce qui devrait être, à savoir le bien, on comprend que soit «historique» le roman du mal, non celui du bien, ou l'utopie du mal et non celle du bien. Bref, une fiction sera dite historique quand elle dépeint le crime, parce qu'elle suit alors la voie allouée à l'histoire, et romanesque ou chimérique quand elle dépeint le bien. Elle sera historique si elle décrit ce qui aurait pu être, à savoir ce type de comportements - les crimes -, dont l'histoire abonde, et ne le sera pas si elle décrit des comportements - sans doute louables -, dont les annales de l'histoire offrent peu d'exemples. On comprend qu'Irodotou fasse précéder sa lecture des deux utopies d'Aline et Valcour d'une lecture d'Isabelle de Bavière. Il nous permet ainsi de comprendre en quoi une fiction peut être désignée par Sade comme «historique».

Mais là n'est pas la grande originalité de ce travail. Irodotou suggère une seconde réponse à cette question, qui explique pourquoi il parle d'«utopies sadiennes». En effet, on aura beau jeu de ne voir dans cette définition du réalisme qu'un parti-pris. Un auteur d'ouvrages de fiction visant à illustrer «les malheurs de la vertu» et «les prospérités du vice» ne saurait que réputer réalistes les récits historiques axés sur les crimes. Mais Sade n'en reste pas là. II impute le lien du désir sexuel et du crime à la nature, accuse la société d'occulter cette vérité dérangeante, et se charge de nous la révéler. Sa «philosophie», écrit-il à sa femme en 1782, c'est que «les mœurs ne dépendent pas de nous», qu' «on n'est pas plus le maître d'adopter dans ces choses-là tel ou tel goût [...] que de se faire brun quand on est roux». ${ }^{6}$ La puissance de la nature est telle que, non contente de frapper d'inanité les institutions censées lui faire obstacle, elle les utilise à son profit. Or, ce rapport de l'institution à la nature induit un autre sens de l'«historicité». Si l'institution nie la nature, elle y succombe à son insu et devient chimérique. Si elle en tient compte, elle est susceptible de la faire évoluer dans un certain sens, certes moralement moins ambitieux, mais plus réaliste.

En effet, s'il ne tenait quaà lui, loin de contrer la nature au moyen des institutions, comme on le voit faire au long de l'histoire, Sade adap- 
terait celles-ci à celle-là. Son manifeste politique, intercalé dans La philosophie dans le boudoir de 1794, promeut un état civil censé combler nos désirs. Certes, on devra y laisser le meurtre impuni, ${ }^{7}$ si bien que la population y décroîtra. Mais, à tout prendre, cette réduction est bénéfique aux républiques, régimes de liberté, et ne contrarie que les monarchies, qui cherchent à accroître leur population pour satisfaire leurs besoins en esclaves. ${ }^{8}$

C'est ici que l'hypothèse d'Irodotou acquiert toute sa valeur. II place, en effet, sa lecture d'Aline et Valcour non seulement sous le signe d'Isabelle de Bavière, qui nous permet de saisir ce qu'est une fiction «historique» pour Sade, mais aussi, et avant tout, sous celui de «Rodrigue ou la Tour Enchantée», un «conte allégorique» publié en 1800 sous le nom de Sade dans Les crimes de l'amour (pp. 21 sqq.), afin de montrer que l'idée d'une histoire de la nature - qui était dans l'air du temps ${ }^{9}$ et allait donner naissance au transformisme lamarckien à partir de 1800 -, n'est pas étrangère à Sade.

C'est ce que lui suggère le déchiffrement de cette allégorie - des fouilles dans la Tour Enchantée, de l'apparition de volcans en éruption et d'animaux gigantesques - à l'aide de Buffon (pp. 33-35). Pour Buffon, il y a une Histoire du monde en ce sens que la nature est «aujourd'hui très différente de ce qu'elle était au commencement», car on ne saurait expliquer autrement l'existence de fossiles et d'ossements d'espèces monstrueuses disparues. ${ }^{10}$ Bref, l'originalité de l'ouvrage d'Iridotou réside, pensons-nous, dans son idée selon laquelle la nature, chez Sade, est «historique» en cet autre sens, apparu à son époque, qu'elle connaît une évolution. ${ }^{11}$ Cette évolution n'est pas à penser, toutefois, comme un changement de nos désirs, mais bien plutôt comme une modification du rapport de l'institution à leur nature. «Les penchants que [l'homme] tient de la nature [...] parvinrent à former en lui, et à son insu, une masse énorme de liens qui le maîtrisent presque partout, sans qu'il s'en aperçoive», dira Lamarck. ${ }^{12}$ Ce que Sade inaugure alors, c'est un discours qui se donne pour tâche de nous révéler cet état de fait, pour nous rendre aptes à produire des institutions qui en tiennent compte.

«En définitive, nous dit Irodotou, les deux épisodes de Butua et Tamoé pourraient se lire comme le double programme d'une narration s'écrivant selon la position d'un commutateur qui permet de choisir le flux de la population. Si l'on pense à sa diminution, on est renvoyé au programme de Butua. Si l'on pense à son maintien et à son efflorescence, on est renvoyé au programme de Tamoé» (p. 125). En effet, si, pour Sade, on ne peut changer la nature humaine, on peut, du moins, édifier des institutions qui la favorisent ou qui essaient de la contrer. Si l'individu n'est pas plus à même d'éradiquer ses passions que de changer la couleur de sa pilosité, il est possible de créer un dispositif institutionnel qui cesse d'exiger hypocritement - leur éradication. Ce dispositif pourrait alors s'attacher à les faire évoluer.

Dès lors, peu importe que La philosophie dans le boudoir prône la communauté des femmes à l'instar de Platon ou Campanella et contrairement à More ou Bacon. Ce qui est nouveau, c'est la raison invoquée en faveur de cette nouvelle institution. Si, comme semble l'indiquer Aline et Valcour dans la lecture d'Irodotou, nous avons le choix entre la communauté des femmes et le mariage, c'est parce que le désir a une historicité au sens d'évolution Irodotou cite cette phrase suggestive d'Aline et Valcour: «Vous aimez encore comme on faisait au dixième siècle» (p. 43). ${ }^{13}$

De ce fait, l'utopie pourra cesser d'être située ailleurs, comme chez Platon, More, Campa- 
nella ou Bacon. Les anciennes «utopies spatiales» seront bientôt remplacées par les «utopies temporelles» - ou «historiques» -, visant à construire de nouvelles institutions. ${ }^{14}$ Irodotou montre ainsi que Sade est à l'orée de ce moment de l'histoire de la pensée où la nature cesse d'être perçue comme une instance autre, que la société aurait loisir d'amener à raison, mais où leur rapport est néanmoins pensé en termes de construction : on peut toujours choisir Butua ou Tamoé. Il s'agit du moment du socialisme utopique. Certes, Fourier optera pour l'amour libre, Owen non, mais l'un et l'autre partiront de l'idée que nul n'est responsable de ses passions, pour proposer des institutions qui les fassent évoluer. ${ }^{15}$

\section{NOTES}

1 Aline et Valcour, ou Le roman philosophique, t. 2 (Paris : Girouard, 1795), 63-234 (wikisource et gallica).

2 Tous les renvois à des pages dans le texte se réfèrent au livre d'Irodotou que nous présentons.

3 Histoire secrète d'Isabelle de Bavière, Reine de France (Paris : Gallimard, 1953), 21 (cité à la p. 58).

4 Comme le pense Sarane Alexandrian, Histoire de la littérature érotique (Paris : Seghers, 1989).

5 Rejetée en son temps par Royer-Collard, médecin de Charenton, et un siècle plus tard par Iwan Bloch, Der Marquis de Sade und seine Zeit (Berlin : Barsdorf, 1900 ; Munich : Heyne, 1978), 450 sqq., cf. 339 sqq.

6 Monsieur le 6 (Paris : Julliard, 1954), 225-226; cité par Maurice Lever, Donatien Alphonse François, marquis de Sade (Paris : Fayard, 1991), 347.
$7 \quad$ La philosophie dans le boudoir (Paris : Gallimard, 1976), 231 sqq.

8 Ibid., 247-248.

9 Notamment chez Maupertuis, Essai sur la formation des corps organisés, 1745. Cf. Jean Ehrard, L'idée de nature en France à l'aube des Lumières (Paris : Flammarion, 1970), 135.

10 Histoire naturelle [choix de textes] (Paris: Gallimard, 1984), 245. Ce texte est de 1778.

11 On sait que Foucault (Les mots et les choses (Paris : Gallimard, 1966)) décèle dans les deux dernières décennies du XVIIIème siècle ce changement de sens de l' «histoire» dans le discours des sciences.

12 Système analytique des connaissances positives de l'homme, 1820, in Jean-Baptiste Lamarck, Pages choisies (Paris : Éditions Sociales, 1957), 56.

13 Aline et Valcour, t. 2, 87.

14 Cf. Richard Saage, Vermessungen des Nirgendwo (Darmstadt : WBG, 1995), 194.

15 Fourier, plus proche de Sade, se propose d'en tenir compte pour infléchir leur marche (Théorie des quatre mouvements (1808), Fuvres complètes, t. 1 (Paris, 1841), 3-21; L'Unité universelle, t. 5 (1822-1823), 153-163; in L'attraction passionnée [choix de textes] (Paris : Pauvert, 1967, 48-49, 185). Robert Owen, plus «constructiviste», espère, en transformant le cadre social, changer la nature humaine elle-même (A New View of Society (1813-1816) (Harmondsworth: Penguin, 1991). 